\title{
Development of Highly Reliable SERS-active Photonic \\ Crystal Fiber Probe and its Application in the Detection of Ovarian Cancer Biomarker in Cyst Fluid
}

Flavien Beffara ${ }^{1,2}$, Jayakumar Perumal' ${ }^{1}$, Aniza Puteri Mahyuddin ${ }^{3}$, Mahesh Choolani' Saif A. Khan ${ }^{4}$, Jean-Louis Auguste ${ }^{2}$, Sylvain Vedraine ${ }^{2}$, Georges Humbert ${ }^{2, *}$, U. S. Dinish $^{1, *}$, Malini Olivo ${ }^{1, *}$

${ }^{1}$ Lab of Bio-Optical Imaging, Singapore Bioimaging Consortium (SBIC), Agency for Science Technology and Research (A*STAR), Singapore

${ }^{2}$ XLIM Research Institute, UMR 7252 CNRS / Limoges University, 123 av. A. Thomas, 87060, Limoges, France

${ }^{3}$ Departments of Obstetrics and Gynecology, Yong Loo Lin School of Medicine, National University of Singapore, Singapore

${ }^{4}$ Department of Chemical and Bimolecular Engineering, National University of Singapore, Singapore

Georges Humbert: georges.humbert@xlim.fr

U. S. Dinish: dinish@sbic.a-star.edu.sg

Malini Olivo: Malini_Olivo@sbic.a-star.edu.sg

* Correspondence to:

\section{Correspondence}

Georges Humbert, XLIM Research Institute, UMR 7252 CNRS / Limoges University, 123 av. A. Thomas, 87060, Limoges, France.

Email: georges.humbert@xlim.fr

U. S. Dinish and Malini Olivo, Lab of Bio-Optical Imaging, Singapore Bioimaging Consortium (SBIC), Agency for Science Technology and Research (A*STAR), Singapore.

Email: dinish@sbic.a-star.edu.sg (U.S.D.) and Malini_Olivo@ sbic.a-star.edu.sg(M.O.)

\section{Abstract}


Conventionally Surface enhanced Raman spectroscopy (SERS) is realized by adsorbing analytes onto nano-roughened planar substrate coated with noble metals (silver or gold) or their colloidal nanoparticles (NPs). Nano scale irregularities in such substrates/NPs could lead to SERS sensors with poor reproducibility and repeatability. Herein, we demonstrate a suspended core photonic crystal fiber (PCF) based SERS sensor with extremely high reproducibility and repeatability in measurement with a relative standard deviation of only $1.5 \%$ and $4.6 \%$ respectively, which makes it more reliable than any existing SERS sensor platforms. In addition, our platform could improve the detection sensitivity owing to the increased interaction area between the guided light and the analyte, which is incorporated into the holes that runs along the length of the PCF. Numerical calculation established the significance of the interplay between light coupling efficiency and evanescent field distribution, which could eventually determine the sensitivity and reliability of the developed SERS active-PCF sensor. As a proof of concept, using this sensor, we demonstrated the detection of haptoglobin, a biomarker for ovarian cancer, contained within the ovarian cyst fluid, which facilitated in differentiating the stages of the cancer. We envision that with necessary refinements, this platform could potentially be translated as a next generation highly sensitive SERS-active opto-fluidic biopsy needle for the detection of biomarkers in body fluids.

Keywords: Surface enhanced Raman scattering, photonic crystal fiber, ovarian cancer, protein sensing, reproducibility and repeatability in measurement, biopsy needle

\section{Introduction}

Raman scattering is a weak inelastic scattering of light by molecules and the resulting 'fingerprint' spectrum consists of narrow peaks, which uniquely represent a specific set of atomic groups/species. The inherent sensitivity limitation of Raman spectroscopy (RS) can be overcome by surface enhanced Raman scattering (SERS) technique, where analyte molecules are adsorbed onto nano-roughened noble metal surfaces or their colloidal nanoparticles (NPs) such as silver or gold (Ag or $\mathrm{Au}$ )[1-7]. Due to plasmonic effect, this leads to a highly sensitive and selective detection platform while the narrow spectral band offers multiplex detection [813]. Even single molecule detection was reported using SERS under certain constraints [4,14]. However, planar plasmonic SERS substrates or NPs responsible for the SERS effect offer interaction between the excitation light and the analyte primarily within the first 10-15 nm near 
the metal surface [15-17]. Moreover, the inherent limitation associated with even the best of lithographic nano-patterning process will result in nano-scale irregularities and that in turn will lead to poor reproducibility and repeatability in measurements. Reproducibility refers to the ability to produce uniform enhanced signal at various spatial locations of the SERS substrate, while, repeatability implies the minimum variation in signal among different batches of substrates. Ideally, a substrate should have uniform nano-scale roughness to yield reproducible signal and possess minimum batch-to-batch variation. Though recent studies demonstrated SERS platforms with signal variation in the range of 5\%-10\% are considered satisfactory [1821], there is a great demand for improved sensitivity and reliability, especially for the detection of biomarkers in clinical bio fluids.

Over the past decade, photonic crystal fibers (PCFs) have been used as SERS platform to improve the excitation area and make sensors more flexible. The well-arranged holes that run along the entire length of the fiber could serve as gas or liquid-chambers leading to an increased interaction area between the guided light and immobilized analyte, resulting in a tremendous enhancement of the Raman signal and thus improved sensitivity [22-28]. Moreover, increasing the interaction area tends to average the obtained signal and therefore nullify the variation in reproducibility and repeatability as in conventional substrates due to the inherent nanoscale irregularities. Among the two classes of PCFs, hollow core PCFs allow direct interaction between the excitation light and the analyte but they suffer from rather narrow transmission bandwidth and spectral shift when they are filled with a liquid analyte [29-31]. On the other hand, solid core PCFs provide fixed and broader transmission bandwidth, even when the fiber is filled with liquid and resulting in stronger Raman signal enhancement [32-35]. In these fibers, the light is guided inside the solid core and the interaction between light and analyte occurs with the evanescent field that overlaps in the cladding.

Haptoglobin (Hp) is a glycoprotein, which is mainly secreted by hepatocytes and usually present in very small quantity in human serum. However, quantity of Hp can increase after a trauma or during an infection or an inflammation. Studies have demonstrated that Hp concentration in patient's serum and fluid within the ovarian cyst is strongly correlated to ovarian cancer [36-39]. Usual methods of detection for ovarian cancer biomarker, which include enzyme-linked immunosorbent assay, electrochemical impedance spectroscopy and chromogen staining are primarily time consuming and quite tedious to perform [40-42]. Though the use of intraoperative frozen section could help in preventing the delay in primary surgery 
[43-45], it has several drawbacks such as variation in accuracy as a result of large cyst size, non-uniformity of the tissue malignancy leading to sampling error and limited availability of staining methods $[45,46]$. Hence, there is an unmet need for a sensitive platform that could be adopted in an intraoperative setting.

To realize a sensitive and reliable SERS biosensor, in this study, for the first time, we demonstrate the record-breaking reproducibility and repeatability in measurement. We developed the SERS sensor using a suspended core PCF (SuC-PCF) with a small triangular silica core surrounded by three large holes. We chose this class of PCF because it enables the fabrication of fibers with a small core surrounded by larges air channels (i.e. air holes) for evanescent field sensing and rapid filling with a liquid or a gas. Numerical simulation helped in identifying the optimal core size for achieving high coupling efficiency and at the same time retaining sufficient evanescent field power for sensing. Finally, as a proof of concept, we detected the levels of Hp in clinical ovarian cyst fluid (OCF) and differentiated the stages of the cancer in several patients. Obtained results correlated well with clinical standards. This study demonstrates a fast, efficient and reliable sensor that allows clinicians to monitor the levels of Hp levels in cyst fluid in order to give the right diagnostic tool for ovarian cancer.

To the best of our knowledge, this is the first demonstration of a sensitive SERS-PCF probe and reliable detection of cancer biomarker in a clinical bio fluid. We envision that such probe could potentially be translated as a highly sensitive biopsy needle for the detection of biomarkers in body fluids, which could pave the way for next generation two-in one sensor for both the sample collection and sensing.

\section{Materials and methods}

\subsection{Fabrication of the SuC-PCF}

The SuC-PCFs were fabricated in XLIM research institute, using the stack-and-draw process. Three capillaries were first assembled in a jacket tube in order to form the design of the SuCPCF. The resulting stack was first drawn to get the fiber preform. After putting the preform in a new jacket tube, it was drawn a second time to get the final fiber. Several fiber samples were fabricated with different core diameters (Dcore $=1.4 \mu \mathrm{m} ; 3.5 \mu \mathrm{m} ; 5 \mu \mathrm{m}$ ) for experimentally 
studying its role on the SERS-sensing performances. We paid special attentions to fabricate the fibers with large air holes for enabling fast liquid infiltrations inside the fibers.

\subsection{Numerical simulation of the coupling efficiency to the SuC-PCF}

We used Comsol Multiphysics and finite element method to simulate the electromagnetic field distribution of the fundamental mode inside a SuC-PCF. The excitation wavelength was set to $633 \mathrm{~nm}$. Refractive indices were 1.457 and 1 for silica and air in the holes respectively. We then computed the coupling efficiency of the light between the fiber and the Raman spectrometer by calculating the overlap integral of the electromagnetic field distributions of the fundamental mode with that of a Gaussian beam as described in [47]. The size of the Gaussian beam was $1.03 \mu \mathrm{m}$ when we used the objective lens with 50x magnification from the Raman spectrometer at our disposal. In order to evaluate the reliability of the coupling efficiency with an operator, the center of the Gaussian beam was randomly positioned 250 times within an area defined by a disk of $0.5 \mu \mathrm{m}$ radius centered on the fiber core.

\subsection{Chemicals}

(3-Aminopropyl)triethoxysilane (APTES), 4-Aminothiophenol (ATP) and Traut's reagent were bought from Sigma-Aldrich. Gold nanoparticles (Au NPs, $60 \mathrm{~nm}$ ) were purchased from BBI Solutions (Redding, CA) and EDTA from BIO-RAD. Phosphate buffered saline (PBS) was obtained from 1st Base and bovine serum albumin (BSA) from PAA Laboratories. Hp antibodies were acquired from Dako Denmark. N-hydroxysuccinimide (NHS) and 1-éthyl-3(3-diméthylaminopropyl)carbodiimide (EDC) were furnished by Thermo Fisher Scientific.

\subsection{Clinical OCF collection}

Clinical OCF samples were collected during surgery. Samples were later transported on ice to the laboratory, centrifuged at $2,000 \times g$ for 10 minutes at $4^{\circ} \mathrm{C}$ and the supernatant were stored at $-80^{\circ} \mathrm{C}$ until analysis. Samples were collected after obtaining written informed consent from each subject and used in accordance with the local ethics committee approved protocol number 2000/00856 (National Healthcare Group, Singapore). 


\subsection{Immobilization of Au NPs inside SuC-PCF}

The following protocol is designed to form a monolayer of anchored NPs inside the fiber holes to make the fiber SERS-active. Initially, the ends of a $10 \mathrm{~cm}$ long SuC-PCF were cleaved and one end was glued inside a $27 \mathrm{G}$ needle and fitted to a syringe pump. The SuC-PCF was then thoroughly cleaned with acetone and dried by repeatedly pumping air inside the fiber holes. $2 \%$ APTES solution (in acetone) was pumped inside the fiber and left to react for $\sim$ fifteen hours to functionalize it to anchor the Au NPs. Subsequently, the SuC-PCF was cleaned with acetone to remove unbound APTES and dried. Later, $60 \mathrm{~nm}$ AuNP solution was injected inside the fiber and left to incubate for 2 hours so that it can bind to previously bound APTES. Finally, the fiber was rinsed with water and dried.

\subsection{Reproducibility and repeatability measurements}

Modal Raman reporter molecule, ATP (1 mM solution) was pumped inside the fiber, which was pre-functionalized with Au NPs, using the syringe pump for $10 \mathrm{~min}$ and the excess of the solution was removed. ATP molecules bound to Au NPs exhibit unique enhanced SERS spectrum with two intense peaks at $1080 \mathrm{~cm}^{-1}$ and $1590 \mathrm{~cm}^{-1}$ corresponding to stretching modes $v \mathrm{CS}, 7 \mathrm{a}$ and $v \mathrm{CC}, 8 \mathrm{a}$, respectively [48-50]. To study the reliability in signal, we monitored the intensity of the $1080 \mathrm{~cm}^{-1}$ peak from ATP. We placed one fiber sensor in the Raman spectrometer and acquired 14 measurements while focusing the light on slightly different locations on the core of the fiber. Though fourteen measurements could not be considered as statistically significant, we believe that it sufficient to prove that our sensor is reproducible. To evaluate the repeatability, we measured the SERS signal from six fibers prepared at different times with the same protocol as in previous case. For each of the six fibers, we took 14 measurements and the intensities presented below are the average of these 14 measurements. Finally, we also tested the response of our sensors to varying concentrations of ATP i.e. $1 \mu \mathrm{M}$, $10 \mu \mathrm{M}, 100 \mu \mathrm{M}$ and $1 \mathrm{mM}$ in order to get a calibration curve. For each concentration of ATP, a fiber sample was prepared following the mentioned protocol and 14 measurements were acquired in order to get an average intensity.

\subsection{Preparation of protein biomarker and antibody-ATP Raman probe for sensing}

Initially, we modified the Hp biomarker in the OCF by adding a sulfhydryl group so that it can bind to previously anchored Au NPs inside the fiber [51-54]. To realize this, the clinical sample 
were incubated with EDTA and Traut's reagent for 30 minutes. Then, the mixture was centrifuged and re-suspended in PBS to remove the excess of reagents.

Next, the SERS readout probe was constructed by attaching ATP Raman reporter molecule to $\mathrm{Hp}$ antibody (Hp-AB). First, we mixed equal volumes of EDC and NHS with $100 \mu \mathrm{lof} \mathrm{Hp}-\mathrm{AB}$ stock solution and left to react for five minutes to activate the carboxylic group in the antibody. Subsequently, we added $10 \mathrm{mM}$ ATP solution and incubated for two hours. This resultant mixture was then centrifuged with a membrane filter to remove the excess of unreacted antibody and ATP.

\subsection{Detection of $\mathrm{Hp}$ in $\mathrm{OCF}$}

Sulfhydryl group modified clinical sample solution (from 2.7) was injected into the fiber and incubated for 30 minutes to allow the thiol-modified $\mathrm{Hp}$ to bind to the already anchored $\mathrm{Au}$ NPs. Subsequently, the fibers were washed with PBS and BSA and then dried. Finally, the solution of AB-ATP probe was incubated for 30 minutes inside the SuC-PCF. The Hp-AB ATP reporter complex will then bind to the immobilized Hp molecules inside the fibers. The fibers were then washed with PBS and water successively and finally dried. After cleaving the free end of fiber, SERS measurements were carried out. Schematic of the protocol is shown in figure 1 (a). The left hand side of schematic represents the syringe pump on which the needles with the glued fibers were attached so that liquids can be pumped inside. The right hand side summarizes the different steps needed to functionalize the SuC-PCF.

\subsection{SERS set up}

The Raman spectra were acquired using Raman spectrometer (Renishaw InVia) operating at $785 \mathrm{~nm}$ laser excitation with a $1200 \mathrm{l} / \mathrm{mm}$ grating. The system was connected to the microscope (Leica) and a $\mathrm{CCD}$ detector cooled at $-70{ }^{\circ} \mathrm{C}$ for signal readout. 50x objective lens $(\mathrm{NA}=0.75$, Leica) coupled the light into the fiber core and collected the emitted Raman signal in backscattering configuration. Figure 1 (b) represents a simplified schematic of this configuration and several views on the functionalized SuC-PCF. The Raman spectrometer was calibrated with the Raman intensity of a silicon standard at $520 \mathrm{~cm}^{-1}$. Baseline correction allowed suppression of the background and the fluorescence band. The integration time was 
$10 \mathrm{~s}$ and no averaging of the signal was done. For the study of reliability, the laser power was $130 \mu \mathrm{W}$ while for the sensor calibration we used $500 \mu \mathrm{W}$. For the sensing of $\mathrm{Hp}$ in OCF, we used $2 \mathrm{~mW}$ laser power.

\section{$2.10 \quad S E M$}

The SEM pictures were taken with a field emission scanning electron microscope (FE-SEM) from JEOL.

\section{Results and discussion}

\subsection{Fiber simulation and characterization}

The simulated designs of the SuC-PCFs with a core diameter of $1.4 \mu \mathrm{m}, 3.5 \mu \mathrm{m}$ and $5 \mu \mathrm{m}$ are shown in figure 2 (a), with the 2-dimensional distribution of the fundamental mode intensity inside a core diameter of $3.5 \mu \mathrm{m}$. In SuC-PCF, the interaction between the excitation laser and the analyte occurs when the evanescent field overlaps in the cladding holes. The fraction of the power inside the hole within $100 \mathrm{~nm}$ from the core surface is more than $1 \%$, allowing strong interactions with the analyte along the fiber length (figure S1, in supporting information, SI). As shown in figure 2 (b-d), it appears that the best coupling efficiency is obtained for a SuCPCF with a $1.4 \mu \mathrm{m}$ core when the light is launched perfectly in the center of the core. In addition, the portion of evanescent field being higher with smaller cores, this fiber or smaller core fibers, could be considered as the best option for SERS sensing, as shown by Oo et al [55]. Practically, however, even by being extremely careful, small misalignments can occur during measurements. In order to increase the reproducibility and repeatability of our sensor, we decided to select a SuC-PCF with a $3.5 \mu \mathrm{m}$ core. Indeed, as shown in figure 2 (b-d), when launching the light with a small offset (i.e. $< \pm 0.5 \mu \mathrm{m}$ ), the fiber with a $3.5 \mu \mathrm{m}$ core becomes the best option available. The average coupling efficiency is higher than the ones of $1.4 \mu \mathrm{m}$ and $5 \mu \mathrm{m}$ core fibers. Numerical simulation helped in analyzing the interplay between the light coupling efficiency (between the fiber core and the objective lens of the Raman spectrometer) and evanescent field distribution in the holes of the PCF. In contrast with previously reported 
works on PCF based SERS sensing [55], this study demonstrates that the optimum fiber core size is not the smallest one, when we consider the measurement reliability criteria.

SEM picture of the cross-section of the fabricated SuC-PCF is available in figure 3 (a). The SuC-PCF exhibits a $3.5 \mu \mathrm{m}$ core diameter and three $\sim 80 \mu \mathrm{m}$ large holes compatible with fast liquid infiltration requirements. The total outer diameter of the fiber is $\sim 200 \mu \mathrm{m}$. Figure 3 (b) shows the anchoring of Au NPs inside the fiber. Unlike SERS substrates, Au NPs need not to be closely and orderly arranged to result in a well reproducible measurement because of the increased interaction surface and resultant averaging effect inside the fiber. Indeed, SuC-PCFs increases the surface area of interaction between excitation light and analyte by few orders of magnitude. This leads to an averaging effect, which in turn corresponds to improved reproducibility. In PCF, the light can interact with much more Au NPs than that in a planar substrate, where the interaction is limited by the laser spot.

\subsection{Evaluation of reproducibility and repeatability}

As shown in figure 4 (a), the fourteen spectra acquired from SuC-PCF (3.5 $\mu \mathrm{m}$ core) were quite similar exhibiting great reproducibility. All measured spectra were repeatable with minimum variation in intensity and relative standard deviation (RSD) was found to be $\sim 1.5 \%$. To the best of our knowledge, this is the highest reproducibility in SERS sensing, where signal variations between 5\%-10\% are common among best ever conventional substrates [18-21]. Detection of biochemicals in the form of liquid and gas (specifically volatile organic compounds) has great relevance in biomedical, chemical, homeland security and environmental monitoring applications and demands very high sensitivity to meet the practically relevant end use applications. Conventional method for these sensing has its own limitations and do not provide the sensitivity in detection for the trace amount of the analyte, especially in an extremely low sample volume. Moreover, early diagnosis of the disease is only possible if the detection of biomarkers is carried out at a very low concentration and often in low sample volume. More often, simultaneous detection of such multiple biomarkers at very low concentration in body fluids is an added challenge even to the commonly used fluorescence based optical modalities. In this context, the reproducibility achieved using our SERS-PCF probe is vital in developing a sensitive biosensor. 
With regards to the repeatability, spectra obtained from six different SuC-PCFs samples are shown in figure 4 (b). All the prominent peaks of ATP are clearly visible with minimal variation. The calculated RSD was found to be $4.6 \%$, which is extremely low in comparison to standard planar substrates/NPs [56]. Our study also revealed that reproducibility and repeatability of SuC-PCF with $1.4 \mu \mathrm{m}$ core is $\sim 5 \%$ and $>15 \%$ respectively, which is in good agreement with our simulation study. In conclusion, the calculated RSDs for reproducibility and repeatability for SuC-PCF sensor with $3.5 \mu \mathrm{m}$ core diameter deeply improves the reliability of SERS-based sensors compared to planar substrates and smaller core SuC-PCF.

High reproducibility and repeatability are critical as most of the SERS sensors are relying on monitoring the intensity of particular spectral peak from an analyte. We also established a calibration curve showing the variations in Raman intensity for different concentrations of ATP. As shown in Figure 4 (c), our sensor exhibits an excellent linearity $\left(R^{2}=0.97779\right)$, which is highly critical while studying the concentration of unknown samples.

\subsection{Detection of $\mathrm{Hp}$ in $\mathrm{OCF}$}

As a proof of concept, we detected $\mathrm{Hp}$ in OCF and its concentration is linked to the different stage of ovarian cancer. As shown in figure 5 (a), the spectrum obtained after incorporating the AB-ATP probe inside the fiber, which is bound to the Hp biomarker, exhibited two characteristic peaks of ATP at $1080 \mathrm{~cm}^{-1}$ and $1590 \mathrm{~cm}^{-1}$, while other reference spectra from bare fiber and after APTES immobilization have no characteristic Raman peaks.

We tested three sets of clinical OCF samples and in first set it is marked as A (benign), B (early cancer) and C (advanced cancer). Figure 5 (b) shows the normalized average Raman intensity obtained from 3 separate SuC-PCFs filled with these three samples. It can be noted that benign OCF exhibited the lowest SERS intensity $(\sim 0.2)$ while OCF ' $C$ ' displayed the highest $(\sim 1)$ whereas ' $\mathrm{B}$ ' with the intermediate value (0.55). $\mathrm{T}$ tests performed between these sets of samples showed that intensities were significantly different $(\mathrm{P}<0.0001$ compared to patient $\mathrm{A})$. To support our results, the concentration of cancer antigen (CA125) in the patients' serum was monitored (Table 1). Concentration of CA125 is generally monitored to determine the malignancy of ovarian cyst and it is considered benign if its value is less than $35 \mathrm{U} / \mathrm{mL}[57,58]$. We could also achieve a similar result from two other sets of OCF samples as shown in figure 5 (c), table 2 and in figure S2, table S1 in SI. Though the exact correlation between these two 
methods need to be further established through clinical study with large set of samples, our initial study showed that SERS active PCF probe could be used for differentiating the stages of ovarian cancer very accurately.

To the best of our knowledge, this is the first demonstration of detection of a cancer biomarker in clinical bio fluid using a sensitive SERS-active PCF probe. The improved reliability (reproducibility and repeatability) of our probe helped in differentiating OCFs accurately. This sensitive platform could be adopted in an intraoperative setting to assess the stage of an ovarian cyst, which will eventually reduce the cost and time involved in current assessment of the disease severity. This will facilitate an appropriate treatment and surgical intervention at very early stage to minimize the risk. We envision that the developed sensor could potentially be translated as a highly sensitive next generation opto-fluidic biopsy needle for the detection of biomarkers in body fluids. Such fiber probes could replace the existing biopsy needle, where biomarker sensing is achieved in a two-step process (sample collection and then detection), while SERS-active PCF probe could serve as two-in one sensor for both the sample collection and sensing. In addition, we envision that such sensor platform could also be used to continuously monitor the concentration of desired biomarkers in critical care units or operating theatres.

\section{Conclusion}

We designed and fabricated a highly reproducible and repeatable SERS-active SuC-PCF probe. Our sensor exhibited record reproducibility and repeatability of $1.5 \%$ and $4.6 \%$ respectively. Numerical study allowed us to select the best core size in regard to the spectrometer at our disposal in order to maximize the coupling efficiency of the light inside the fiber and the evanescent field. As a proof of concept, the detection of Hp in OCF was successfully carried out using this highly reliable SERS active SuC-PCF probe. Moreover, we were able to differentiate the stages of ovarian cancer by correlating it to the concentration of $\mathrm{Hp}$ in OCF and our SERS results showed excellent correlation with standard clinical method for the detection of ovarian cancer. A large-scale statistically significant clinical study is in progress and that would allow us in establishing the normalized cut-off SERS intensity to differentiate benign and early cancer. We are currently investigating on effective ways to attach the antibody inside the fiber prior to the incorporation of the clinical fluid, which will improve the practical 
usability of the sensor. We envision that this will pave the way for a new generation of SERSactive PCF probe with ultra-high sensitivity that would allow reliable detections of infinitesimal amount of biomarkers to facilitate early disease diagnosis.

\section{Acknowledgements}

Authors sincerely appreciate the funding support from Biomedical Research Council (BMRC), Agency for Science Technology and Research (A*STAR), Singapore and PICS project, "Biosensing platforms based on specialty optical fibers as practical clinical diagnostic tools" from CNRS, France. 


\section{References $[$ [FB1]}

1. M. G. Albrecht and J. A. Creighton, "Anomalously intense Raman spectra of pyridine at a silver electrode," Journal of the American Chemical Society 99(15), 5215-5217 (1977).

2. D. L. Jeanmaire and R. P. V. Duyne, J. Electroanal. Chem. 1977, 84, 1.

3. M. Moskovits, "Surface-enhanced Raman spectroscopy: a brief retrospective," Journal of Raman Spectroscopy 36(6-7), 485-496 (2005).

4. S. Nie, "Probing Single Molecules and Single Nanoparticles by Surface-Enhanced Raman Scattering," Science 275(5303), 1102-1106 (1997).

5. H. Ko, S. Singamaneni, and V. V. Tsukruk, "Nanostructured Surfaces and Assemblies as SERS Media," Small 4(10), 1576-1599 (2008).

6. C. Zhang, S. Z. Jiang, Y. Y. Huo, A. H. Liu, S. C. Xu, X. Y. Liu, Z. C. Sun, Y. Y. Xu, Z. Li, and B. Y. Man, "SERS detection of R6G based on a novel graphene oxide/silver nanoparticles/silicon pyramid arrays structure," Opt. Express 23(19), 24811 (2015).

7. C. Zhang, C. Li, J. Yu, S. Jiang, S. Xu, C. Yang, Y. J. Liu, X. Gao, A. Liu, and B. Man, "SERS activated platform with three-dimensional hot spots and tunable nanometer gap," Sensors and Actuators B: Chemical 258, 163-171 (2018).

8. S. P. Ravindranath, Y. Wang, and J. Irudayaraj, "SERS driven cross-platform based multiplex pathogen detection," Sensors and Actuators B: Chemical 152(2), 183-190 (2011).

9. K. K. Maiti, A. Samanta, M. Vendrell, K.-S. Soh, M. Olivo, and Y.-T. Chang, "Multiplex cancer cell detection by SERS nanotags with cyanine and triphenylmethine Raman reporters," Chem. Commun. 47(12), 3514-3516 (2011).

10. H. Kang, S. Jeong, Y. Park, J. Yim, B.-H. Jun, S. Kyeong, J.-K. Yang, G. Kim, S. Hong, L. P. Lee, J.-H. Kim, H.-Y. Lee, D. H. Jeong, and Y.-S. Lee, "Near-Infrared SERS Nanoprobes with Plasmonic Au/Ag Hollow-Shell Assemblies for In Vivo Multiplex Detection," Advanced Functional Materials 23(30), 3719-3727 (2013).

11. U. S. Dinish, G. Balasundaram, Y.-T. Chang, and M. Olivo, "Actively Targeted In Vivo Multiplex Detection of Intrinsic Cancer Biomarkers Using Biocompatible SERS Nanotags," Scientific Reports 4, 4075 (2014).

12. U. S. Dinish, G. Balasundaram, Y. T. Chang, and M. Olivo, "Sensitive multiplex detection of serological liver cancer biomarkers using SERS-active photonic crystal fiber probe: SERS active photonic crystal fiber probe for multiplex biomarker detection," Journal of Biophotonics 7(1112), 956-965 (2014).

13. J. Xu, C. Li, H. Si, X. Zhao, L. Wang, S. Jiang, D. Wei, J. Yu, X. Xiu, and C. Zhang, "3D SERS substrate based on Au-Ag bi-metal nanoparticles/MoS 2 hybrid with pyramid structure," Opt. Express 26(17), 21546 (2018).

14. K. Kneipp, Y. Wang, H. Kneipp, L. T. Perelman, I. Itzkan, R. R. Dasari, and M. S. Feld, "Single Molecule Detection Using Surface-Enhanced Raman Scattering (SERS)," Phys. Rev. Lett. 78(9), 1667-1670 (1997).

15. G. J. Kovacs, R. O. Loutfy, P. S. Vincett, C. Jennings, and R. Aroca, "Distance dependence of SERS enhancement factor from Langmuir-Blodgett monolayers on metal island films: evidence for the electromagnetic mechanism," Langmuir 2(6), 689-694 (1986).

16. G. Chumanov, K. Sokolov, B. W. Gregory, and T. M. Cotton, "Colloidal metal films as a substrate for surface-enhanced spectroscopy," J. Phys. Chem. 99(23), 9466-9471 (1995).

17. J. P. Camden, J. A. Dieringer, J. Zhao, and R. P. Van Duyne, "Controlled Plasmonic Nanostructures for Surface-Enhanced Spectroscopy and Sensing," Acc. Chem. Res. 41(12), 1653-1661 (2008).

18. L. Wu, W. Wang, W. Zhang, H. Su, Q. Liu, J. Gu, T. Deng, and D. Zhang, "Highly sensitive, reproducible and uniform SERS substrates with a high density of three-dimensionally distributed hotspots: gyroid-structured Au periodic metallic materials," Npg Asia Materials 10, e462 (2018).

19. S. Jiang, J. Guo, C. Zhang, C. Li, M. Wang, Z. Li, S. Gao, P. Chen, H. Si, and S. Xu, "A sensitive, uniform, reproducible and stable SERS substrate has been presented based on MoS2@Ag nanoparticles@ pyramidal silicon," RSC Adv. 7(10), 5764-5773 (2017). 
20. Y. Jin, Y. Wang, M. Chen, X. Xiao, T. Zhang, J. Wang, K. Jiang, S. Fan, and Q. Li, "Highly Sensitive, Uniform, and Reproducible Surface-Enhanced Raman Spectroscopy Substrate with Nanometer-Scale Quasi-periodic Nanostructures," ACS Appl. Mater. Interfaces 9(37), 3236932376 (2017).

21. K. R. Strehle, D. Cialla, P. Rösch, T. Henkel, M. Köhler, and J. Popp, "A Reproducible SurfaceEnhanced Raman Spectroscopy Approach. Online SERS Measurements in a Segmented Microfluidic System," Anal. Chem. 79(4), 1542-1547 (2007).

22. U. S. Dinish, F. Beffara, G. Humbert, J.-L. Auguste, and M. Olivo, "Surface-enhanced Raman scattering-active photonic crystal fiber probe: Towards next generation liquid biopsy sensor with ultra high sensitivity," Journal of Biophotonics 12(11), e201900027 (2019).

23. N. Zhang, G. Humbert, T. Gong, P. P. Shum, K. Li, J.-L. Auguste, Z. Wu, D. J. J. Hu, F. Luan, Q. X. Dinh, M. Olivo, and L. Wei, "Side-channel photonic crystal fiber for surface enhanced Raman scattering sensing," Sensors and Actuators B: Chemical 223, 195-201 (2016).

24. Y. Guo, M. K. Khaing Oo, K. Reddy, and X. Fan, "Ultrasensitive Optofluidic Surface-Enhanced Raman Scattering Detection with Flow-through Multihole Capillaries," ACS Nano 6(1), 381388 (2012).

25. Y. Zhang, C. Shi, C. Gu, L. Seballos, and J. Z. Zhang, "Liquid core photonic crystal fiber sensor based on surface enhanced Raman scattering," Applied Physics Letters 90(19), 193504 (2007).

26. A. Khetani, V. S. Tiwari, A. Momenpour, and H. Anis, "Monitoring of adenosine within hollow core photonic crystal fiber by surface enhanced Raman scattering (SERS)," in 2011 11th IEEE International Conference on Nanotechnology (2011), pp. 973-977.

27. A. Khetani, A. Momenpour, E. I. Alarcon, and H. Anis, "Hollow core photonic crystal fiber for monitoring leukemia cells using surface enhanced Raman scattering (SERS)," Biomedical Optics Express 6(11), 4599 (2015).

28. Y. Han, S. Tan, M. K. K. Oo, D. Pristinski, S. Sukhishvili, and H. Du, "Towards Full-Length Accumulative Surface-Enhanced Raman Scattering-Active Photonic Crystal Fibers," Advanced Materials 22(24), 2647-2651 (2010).

29. G. Antonopoulos, F. Benabid, T. A. Birks, D. M. Bird, J. C. Knight, and P. S. J. Russell, "Experimental demonstration of the frequency shift of bandgaps in photonic crystal fibers due to refractive index scaling," Optics Express 14(7), 3000-3006 (2006).

30. A. Khetani, V. S. Tiwari, A. Harb, and H. Anis, "Monitoring of heparin concentration in serum by Raman spectroscopy within hollow core photonic crystal fiber," Optics Express 19(16), 15244 (2011).

31. V. S. Tiwari, A. Khetani, M. Naji, and H. Anis, "Study of surface enhanced Raman scattering (SERS) within hollow core photonic crystal fiber," in 2009 IEEE Sensors (IEEE, 2009), pp. 367-370.

32. J. C. Knight, T. A. Birks, P. S. J. Russell, and D. M. Atkin, "All-silica single-mode optical fiber with photonic crystal cladding," Optics Letters 21(19), 1547- (1996).

33. T. A. Birks, J. C. Knight, and P. S. J. Russell, "Endlessly single-mode photonic crystal fiber," Optics Letters 22(13), 961- (1997).

34. M. K. K. Oo, Y. Han, R. Martini, S. Sukhishvili, and H. Du, "Forward-propagating surfaceenhanced Raman scattering and intensity distribution in photonic crystal fiber with immobilized Ag nanoparticles," Opt. Lett. 34(7), 968 (2009).

35. T. Gong, Y. Cui, D. Goh, K. K. Voon, P. P. Shum, G. Humbert, J.-L. Auguste, X.-Q. Dinh, K.-T. Yong, and M. Olivo, "Highly sensitive SERS detection and quantification of sialic acid on single cell using photonic-crystal fiber with gold nanoparticles," Biosensors and Bioelectronics 64, 227-233 (2015).

36. A. Mahyuddin, L. Liu, C. Zhao, N. Kothandaraman, M. Salto-Tellez, B. Pang, D. Lim, L. Annalamai, J. Chan, T. Lim, A. Biswas, G. Rice, K. Razvi, and M. Choolani, "Diagnostic accuracy of haptoglobin within ovarian cyst fluid as a potential point-of-care test for epithelial ovarian cancer: an observational study," BJOG: An International Journal of Obstetrics \& Gynaecology 125(4), 421-431 (2018).

37. J. Perumal, A. Mahyuddin, G. Balasundaram, D. Goh, C. Y. Fu, A. Kazakeviciute, U. Dinish, M. Choolani, and M. Olivo, "SERS-based detection of haptoglobin in ovarian cyst fluid as a point- 
of-care diagnostic assay for epithelial ovarian cancer," Cancer Management and Research Volume 11, 1115-1124 (2019).

38. J. Perumal, G. Balasundaram, A. P. Mahyuddin, M. Choolani, and M. Olivo, "SERS-based quantitative detection of ovarian cancer prognostic factor haptoglobin," International journal of nanomedicine 10, 1831-1840 (2015).

39. C. Zhao, L. Annamalai, C. Guo, N. Kothandaraman, S. C. L. Koh, H. Zhang, A. Biswas, and M. Choolani, "Circulating haptoglobin is an independent prognostic factor in the sera of patients with epithelial ovarian cancer," Neoplasia (New York, N.Y.) 9(1), 1-7 (2007).

40. T. Porstmann and S. T. Kiessig, "Enzyme immunoassay techniques an overview," Journal of Immunological Methods 150(1), 5-21 (1992).

41. Y.-C. C. Liu, N. Rieben, L. Iversen, B. S. Sørensen, J. Park, J. Nygård, and K. L. Martinez, "Specific and reversible immobilization of histidine-tagged proteins on functionalized silicon nanowires," Nanotechnology 21(24), 245105 (2010).

42. T.-M. Cheng, J.-P. Pan, S.-T. Lai, L.-P. Kao, H.-H. Lin, and S. J. T. Mao, "Immunochemical property of human haptoglobin phenotypes: Determination of plasma haptoglobin using typematched standards," Clinical Biochemistry 40(13), 1045-1056 (2007).

43. P. Cross, R. Naik, A. Patel, A. Nayar, J. Hemming, S. Williamson, J. Henry, R. Edmondson, K. Godfrey, K. Galaal, A. Kucukmetin, and A. Lopes, "Intra-operative frozen section analysis for suspected early-stage ovarian cancer: 11 years of Gateshead Cancer Centre experience," BJOG: An International Journal of Obstetrics \& Gynaecology 119(2), 194-201 (2012).

44. P. Baker and E. Oliva, "A Practical Approach to Intraoperative Consultation in Gynecological Pathology," International Journal of Gynecological Pathology 27(3), 353-365 (2008).

45. N. D. Ratnavelu, A. P. Brown, S. Mallett, R. J. Scholten, A. Patel, C. Founta, K. Galaal, P. Cross, and R. Naik, "Intraoperative frozen section analysis for the diagnosis of early stage ovarian cancer in suspicious pelvic masses," Cochrane Database of Systematic Reviews (2016).

46. P. M. A. J. Geomini, L. D. Zuurendonk, G. L. Bremer, J. de Graaff, R. F. P. M. Kruitwagen, and B. W. J. Mol, "The impact of size of the adnexal mass on the accuracy of frozen section diagnosis," Gynecologic Oncology 99(2), 362-366 (2005).

47. N. S. Kapany and J. J. Burke, "Waveguide Mode Lauching," in Optical Waveguides, Quantum Electronics-Principles and Applications (Academic Press, 1972), pp. 159-179.

48. M. Osawa, N. Matsuda, K. Yoshii, and I. Uchida, "Charge transfer resonance Raman process in surface-enhanced Raman scattering from p-aminothiophenol adsorbed on silver: Herzberg-Teller contribution," J. Phys. Chem. 98(48), 12702-12707 (1994).

49. J. Zheng, Y. Zhou, X. Li, Y. Ji, T. Lu, and R. Gu, "Surface-Enhanced Raman Scattering of 4Aminothiophenol in Assemblies of Nanosized Particles and the Macroscopic Surface of Silver," Langmuir 19(3), 632-636 (2003).

50. K. Uetsuki, P. Verma, T. Yano, Y. Saito, T. Ichimura, and S. Kawata, "Experimental Identification of Chemical Effects in Surface Enhanced Raman Scattering of 4Aminothiophenol," J. Phys. Chem. C 114(16), 7515-7520 (2010).

51. Cao, R. Jin, and C. A. Mirkin, "DNA-Modified Core-Shell Ag/Au Nanoparticles," J. Am. Chem. Soc. 123(32), 7961-7962 (2001).

52. L. Carlini, C. Fasolato, P. Postorino, I. Fratoddi, I. Venditti, G. Testa, and C. Battocchio, "Comparison between silver and gold nanoparticles stabilized with negatively charged hydrophilic thiols: SR-XPS and SERS as probes for structural differences and similarities," Colloids and Surfaces A: Physicochemical and Engineering Aspects 532, 183-188 (2017).

53. J. J. Storhoff, S. S. Marla, P. Bao, S. Hagenow, H. Mehta, A. Lucas, V. Garimella, T. Patno, W. Buckingham, W. Cork, and U. R. Müller, "Gold nanoparticle-based detection of genomic DNA targets on microarrays using a novel optical detection system," Biosensors and Bioelectronics 19(8), 875-883 (2004).

54. Y. Xue, X. Li, H. Li, and W. Zhang, "Quantifying thiol-gold interactions towards the efficient strength control," Nature Communications 5, 4348 (2014).

55. M. K. Khaing Oo, Y. Han, J. Kanka, S. Sukhishvili, and H. Du, "Structure fits the purpose: photonic crystal fibers for evanescent-field surface-enhanced Raman spectroscopy," Optics Letters 35(4), 466 (2010). 
56. M. J. Natan, "Concluding Remarks Surface enhanced Raman scattering," Faraday Discuss. 132(0), 321-328 (2006).

57. I. JACOBS, D. ORAM, J. FAIRBANKS, J. TURNER, C. FROST, and J. G. GRUDZINSKAS, "A risk of malignancy index incorporating CA 125, ultrasound and menopausal status for the accurate preoperative diagnosis of ovarian cancer," BJOG: An International Journal of Obstetrics \& Gynaecology 97(10), 922-929 (1990).

58. E. P. Diamandis, H. A. Fritche, H. Lilja, D. W. Chan, and M. K. Schwartz, "Tumor Markers: Physiology, Pathobiology, Technology and Clinical Applications," in Tumor Markers:

Physiology, Pathobiology, Technology and Clinical Applications, AACC Press (2002), pp. 239252. 


\section{Table}

\begin{tabular}{|c|c|c|c|}
\hline Patient & Tumor condition & CA125 (U/mL) & Histology \\
\hline A & Benign & 11 & $\begin{array}{c}\text { Mucinous } \\
\text { cystadenoma }\end{array}$ \\
\hline B & Malignant (Early) & 45.9 & $\begin{array}{c}\text { Serous } \\
\text { adenocarcinoma }\end{array}$ \\
\hline C & Malignant (Advanced) & 133.3 & Clear cell carcinoma \\
\hline
\end{tabular}

Table 1: Histology and CA 125 results from set 1 of OCF samples

\begin{tabular}{|c|c|c|c|}
\hline Patient & Tumor condition & CA125 $(\mathbf{U} / \mathbf{m L})$ & Histology \\
\hline D & Benign & 9.1 & Serous cystadenoma \\
\hline E & Malignant (Advanced) & 3283 & Serous carcinoma \\
\hline
\end{tabular}

Table 2: Histology and CA 125 results from set 2 of OCF samples 


\section{Figures}

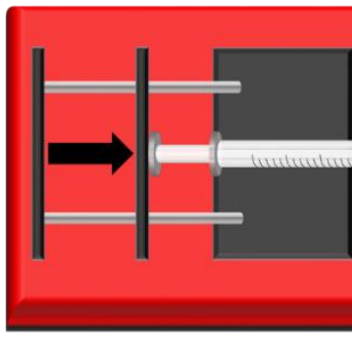

(b) (a)

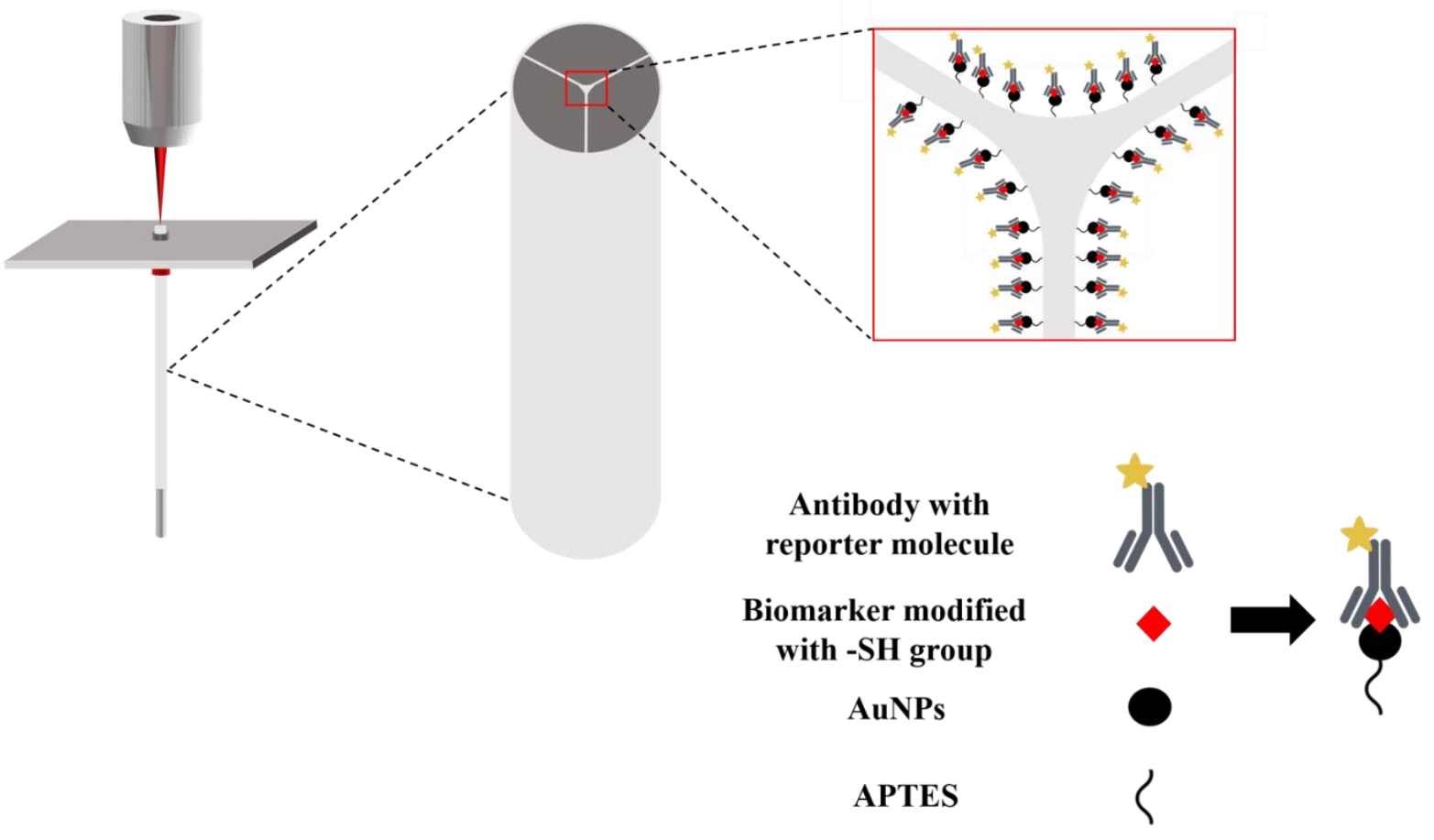

Figure 1 (a) Schematic of the functionalization process inside the holes of the PCF for biomarker sensing. Fiber is connected to a syringe needle. (b) Coupling of PCF to objective lens of Raman spectrometer and backscattering configuration of the fiber for signal collection. Zoom in on the fiber end face and holes with the attached protein and read out Raman tag. 
(a)
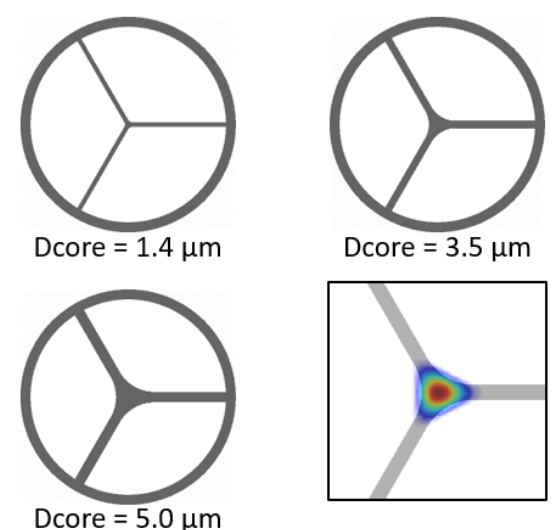

(c)

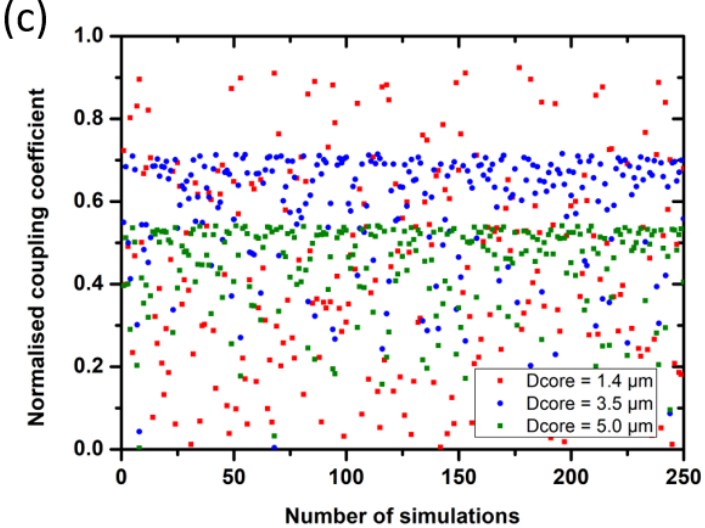

(b)

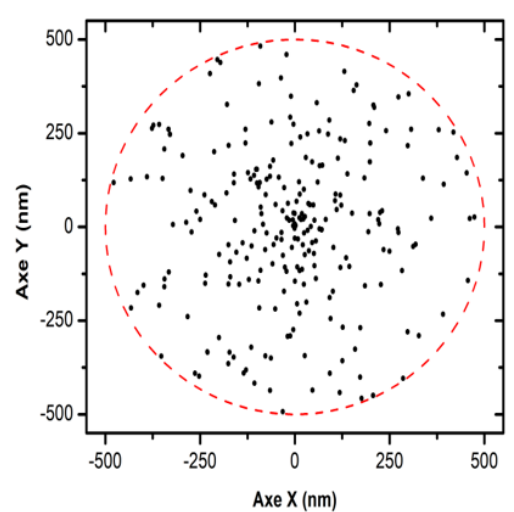

(d)

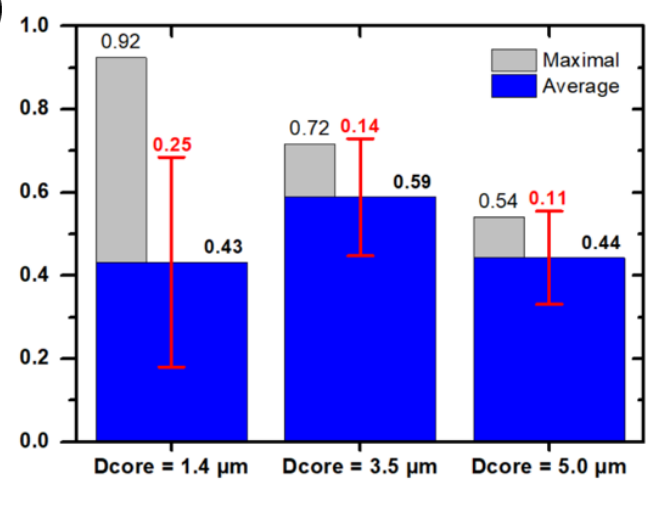

Figure 2 (a) Design of the simulated SuC-PCFs with different core sizes, and zoom-in on the $2 D$ distribution of thePoynting vector of the fundamental mode propagated in a core diameter of $3.5 \mu \mathrm{m}$ (superimposed in light grey). (b) Distribution of the center of a Gaussian beam (corresponding to the laser beam from the Raman spectrometer) randomly positioned (250 times) within an area limited by a circle of $0.5 \mu \mathrm{m}$ radius around the center of the fiber. (c) Normalized coupling coefficients between the Gaussian beam (following the positions in $(b)$ ) and the fundamental mode of SuC-PCFs with different core sizes. (d) Calculated maximum, and average coupling coefficient values from the values plotted in (c). Standard deviation in average coupling coefficient is denoted with red lines. 
(a)

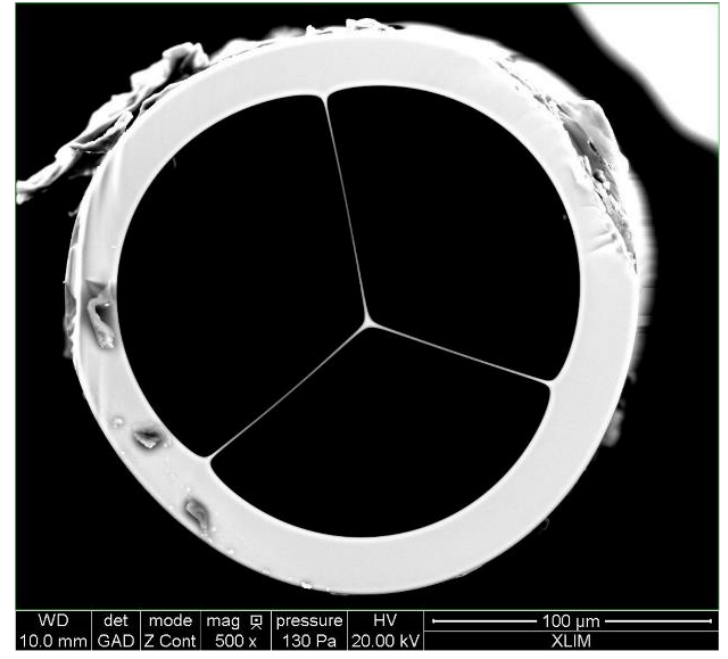

(b)

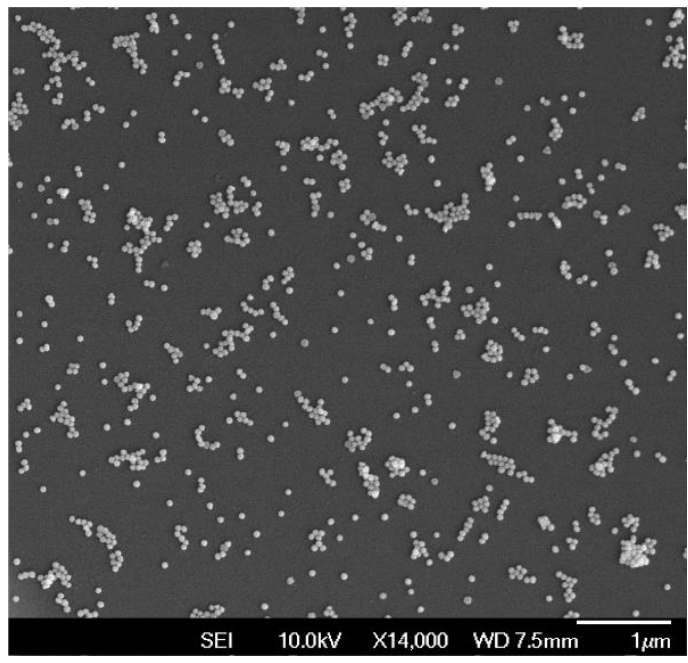

Figure 3 (a) SEM picture of the end face of SuC-PCF. (b) SEM pictures of the anchored Au NPs inside the fiber. 
(a)

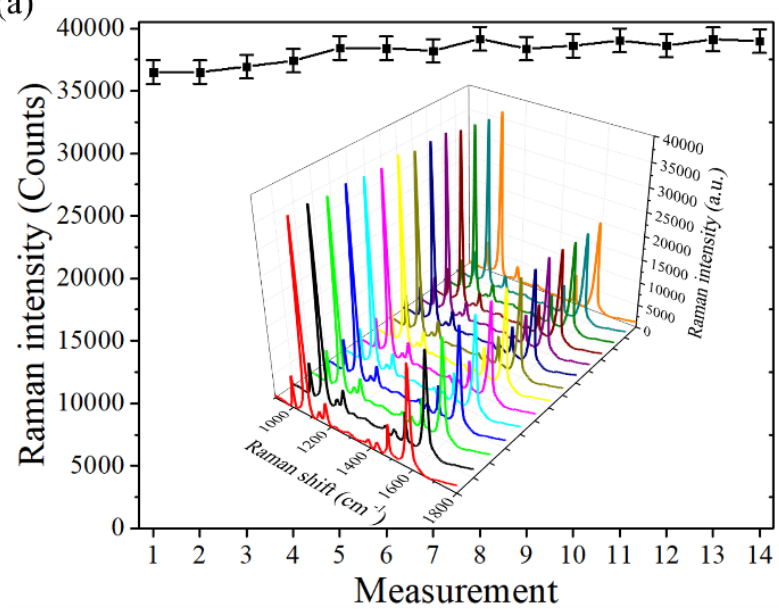

(b)

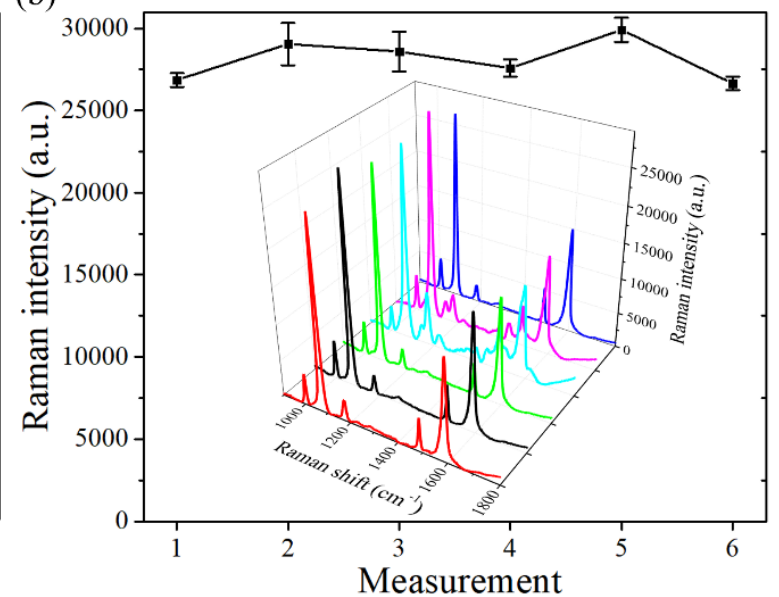

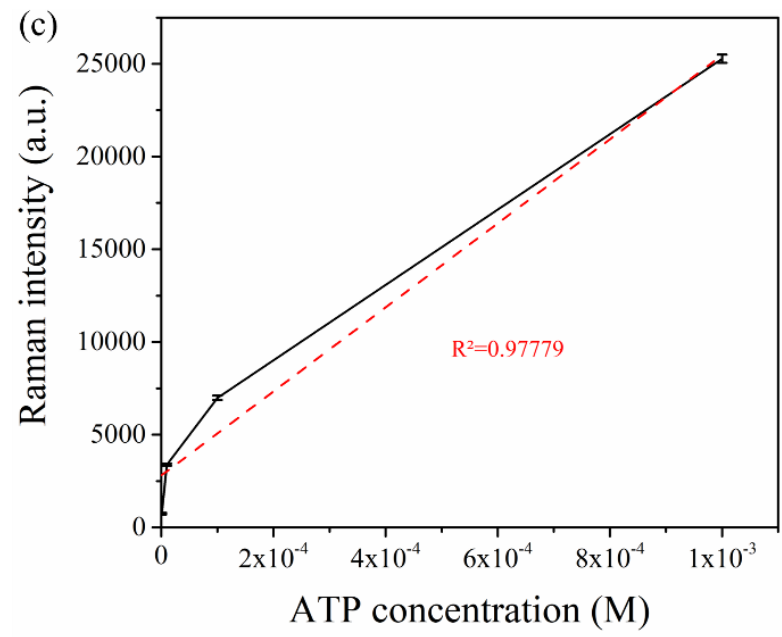

Figure 4 (a) Variations in the Raman intensity of $1080 \mathrm{~cm}^{-1}$ peak (14 measurements) from the SuC-PCF showing the excellent reproducibility of the sensor, error bars represent the standard deviation of the 14 measurements. Acquired 14 spectra from one of the representative fiber are shown. (b) Variations in the Raman intensity of the $1080 \mathrm{~cm}^{-1}$ peak from 6 different fibers showing the good repeatability, error bars represent the standard deviations of 14 measurements acquired for each fiber; Individual spectrum from 6 different fibers are shown. (c) Calibration curve of Raman intensity with ATP concentration showing the good linearity of the sensor, error bars represent the standard deviation obtained from 14 measurements. 

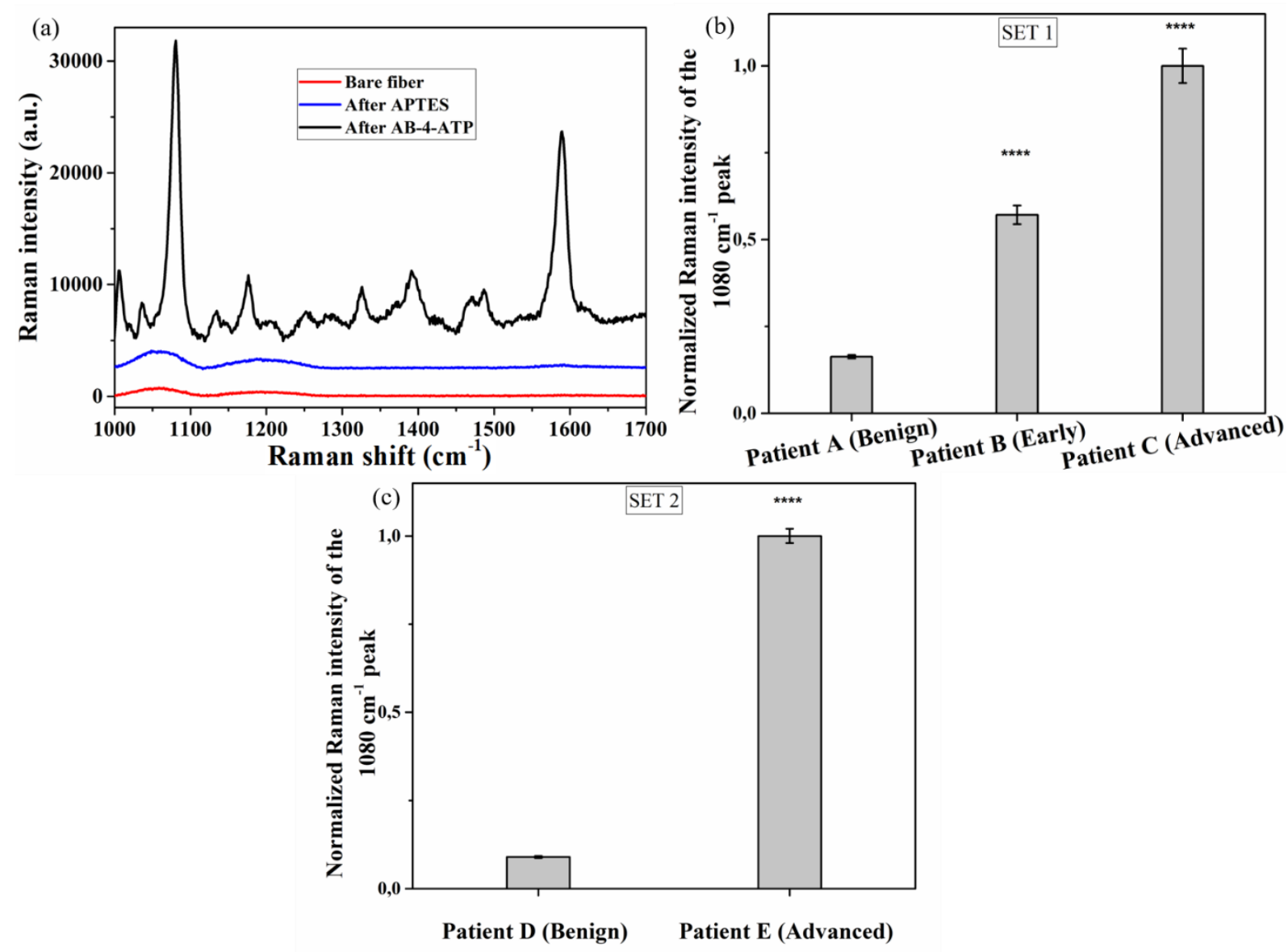

Figure 5 (a) SERS spectra of the bare fiber, the fiber after anchoring with APTES and after adding AB-ATP that binds to Hp biomarker demonstrating the specificity. All prominent peaks of ATP are observed. (b) Normalized Raman intensity of the $1080 \mathrm{~cm}^{-1}$ peak from clinical OCF having different stages of cancer. $* * * * P<0.0001$ denotes significance when compared to Patient A. (c) Normalized Raman intensity of the $1080 \mathrm{~cm}^{-1}$ peak from the second set of clinical OCF depicting different stages of cancer. $* * * * P<0.0001$ denotes significance when compared to Patient D. 